\title{
Communication impairment in patients with Parkinson's disease: challenges and solutions
}

This article was published in the following Dove Press journal:

Journal of Parkinsonism and Restless Legs Syndrome

23 May 2016

Number of times this article has been viewed

\section{Thomas Holtgraves Chelsea Cadle}

Department of Psychological Science, Ball State University, Muncie, IN, USA

Correspondence: Thomas Holtgraves Department of Psychological Science, Ball State University, Muncie, IN 47306 USA

Email 00t0holtgrav@bsu.edu

\begin{abstract}
Parkinson's disease (PD) is viewed primarily as a motor disorder. However, recent researches suggest that there is also a variety of communication deficits associated with this disorder. In this paper, we review some of these researches and provide a set of recommendations designed to improve communicative outcomes when interacting with people who have PD. A variety of comprehension deficits have been documented in PD, including syntactic, pragmatic, and semantic deficits, as well as an impaired ability to recognize emotions. People with PD are also impaired in terms of language production, possibly in part because of their comprehension deficits. Major production deficits include reduced informational content, longer and more frequent pauses and associated turn-taking disruption, inappropriate levels of politeness, and deficits in various nonverbal accompaniments. Awareness of these deficits, and simple, common sense communicative adjustments, can greatly improve communication with people with PD.

Keywords: Parkinson's disease, communication recommendations, pragmatics, comprehension deficits, production deficits, nonliteral language, politeness
\end{abstract}

\section{Introduction}

Parkinson's disease (PD) is currently the second most common neurodegenerative disorder, showing a prevalence rate of $0 \%-3 \%$ of the entire population in industrialized countries and $\sim 1 \%$ in people older than 60 years. ${ }^{1}$ Although PD has traditionally been classified as a paradigmatic movement disorder characterized by muscle rigidity, bradykinesia, resting tremor, and postural instability, recent research has revealed a broad spectrum of nonmotor symptoms, including cognitive deficits (short-term memory, executive functions [EFs]), and language impairment. ${ }^{2}$ Although each individual may experience PD symptoms in varying degrees, it is now well established that language and communication deficits appear early on in the disease progression. As effective communication is critical to developing and maintaining relationships, it is important for people with PD and their caretakers/physicians to be aware of changes in communication abilities that might occur.

Increased understanding of the communication impairments and associated cognitive and neural mechanisms stands to benefit both individuals with PD and their physicians/caretakers. Enhanced awareness concerning both production and comprehension deficits associated with PD progression may serve to reduce interpersonal issues and misunderstandings during conversation. Furthermore, implementing the recommendations suggested in this review may reduce communication deficiencies and help improve overall quality of life. In this paper, we review researches on a range 
of communication deficits and associated cognitive and neural mechanisms. We first review what is known about comprehension deficits in PD, followed by a consideration of production deficits. At the end of each section, we provide a set of recommendations based on the empirical findings. A summary of our recommendations is provided in Table 1.

\section{Comprehension deficits}

There has been a considerable amount of research examining the extent to which PD is associated with various comprehension deficiencies and associated cognitive and neural processes that may contribute to those deficits. In general, this research can be organized around syntactic comprehension, emotion recognition, and pragmatic comprehension (ie, recognition of speaker meaning).

\section{Syntactic comprehension deficits}

The role of syntactic comprehension is crucial in normal language understanding. Successful processing of syntactic information allows people to extract meaning from utterances by combining words in a particular order and rejecting unlikely word arrangements. Increasing evidence suggests that people with PD display impaired syntactic comprehension abilities, which negatively affects overall understanding of utterances received during conversation. Friederici et $\mathrm{al}^{3}$ studied nondemented PD patients, all of whom were taking medication and were not suffering from depression. Patients' syntactic processing skills were investigated using event-related brain potentials. The results suggested that, while early semantic processing appears to remain normal, late-stage syntactic integration is impaired. ${ }^{3}$ Such findings

Table I Summary of recommendations based on documented communication deficits in Parkinson's disease

\begin{tabular}{lll}
\hline Deficits & Recommendation & $\begin{array}{l}\text { Representative } \\
\text { study }\end{array}$ \\
\hline Comprehension deficits & \\
I & Avoid complex syntax & 3,25 \\
2 & Avoid taxing working memory & $4-6,18,26$ \\
3 & Clearly delineate topics & 7 \\
4 & Avoid nonliteral meaning & $19-23$ \\
5 & Use performative verbs & 21 \\
6 & Avoid reliance on emotional expression & $12-17$ \\
7 & Minimize distractions & 7 \\
Production deficits & \\
I & Do not judge & 2 \\
2 & Be patient/do not rush & $26-28,42$ \\
3 & Ask questions regarding utterance & $29,30,51$ \\
& comprehension & $34-37,48$ \\
4 & Ask simple questions & $36,42,43$ \\
\hline
\end{tabular}

support the earlier work performed by Grossman et al, ${ }^{4,5}$ which suggested that medicated PD patients without dementia consistently suffer from comprehension difficulties when presented with syntactically complex sentences. Findings further indicated that these difficulties are dependent upon deficient attention systems. ${ }^{4}$ In attempting to delineate what characterizes a "complex" sentence that PD patients may struggle to comprehend, Grossman et $\mathrm{al}^{6}$ monitored cerebral bloodoxygen-level dependent activity with functional magnetic resonance imaging, while PD patients were presented with sentences differing in their grammatical structure. Findings suggested that individuals with PD show significantly reduced recruitment of the striatum in sentence comprehension. ${ }^{6}$ In the frontal-striatal-thalamic loop, the striatum is understood to function as a cognitive resource for working memory (WM) and information-processing speed. Taken together, studies by Grossman et $\mathrm{al}^{4-6}$ suggest that people with PD struggle to comprehend sentences that are grammatically structured with an objective-relevant clause and have difficulty with utterances that impose increased short-term memory demands resulting from long antecedent-gap linkages.

Findings from the studies of Grossman et $\mathrm{al}^{4-6}$ implicate impaired executive processes as possible sources for language comprehension deficits that result from PD. Indeed, several studies suggest that various comprehension deficits may be attributable to certain cognitive deficits, such as the role of WM in syntactic processing. Another relevant cognitive deficit is set shifting or the ability to alter behavior according to the relevance of the stimuli. A review of this area by Dirnberger and Jahanshahi ${ }^{7}$ contained several interesting conclusions about EFs in PD and set shifting in particular. According to these authors, attention deficits in PD occur primarily for tasks involving internally generated cues, such as the Wisconsin Card Sorting Test; when an external cue is available, attention deficits for PD relative to control participants are minimized..$^{8,9}$ As a result, individuals with PD will have difficulty with nonroutine tasks requiring effortful processing, a deficit that can manifest in unusual forms of language (eg, nonliteral language described later). Moreover, this internal cue processing deficit likely underlies the difficulty PD individuals have with planning (ie, internal cues are required) as evidenced by their inferior performance on the Tower of London and related tasks, ${ }^{10}$ a deficit that can impact language production as described later.

\section{Impaired emotion recognition in PD}

The ability to infer the emotional states of others is made possible through accurate recognition of facial expressions ${ }^{11}$ 
and correct interpretation of implied meaning through tone of voice. Assessing these nonverbal cues often reveals what a speaker is truly attempting to accomplish by allowing the listener to qualify the explicit meaning of a sentence. Though the literature is inconsistent, several findings of studies suggest that emotion recognition abilities, utilizing facial expression or speech prosody are impaired in people with PD. Dewick et al ${ }^{12}$ investigated a variety of face processing skills in a group of idiopathic, nondemented PD patients, all of whom were taking anti-Parkinsonian medication at the time the study was conducted. Face processing skills assessed during the investigation included: 1) the ability to match unfamiliar faces by their identity; 2) discriminate faces by sex or emotional expression; and 3) discriminate faces according to which speech sound was being produced. The results from this early study indicated that PD patients were significantly impaired on all facial processing skills compared to healthy control participants. ${ }^{12}$ This study led to an early conclusion that PD causes a basic deficit in encoding facial expression, which was then suggested to cause impaired processing for all later interpretation stages. However, additional research investigating similar nonverbal communication deficits suggests that not all individuals with PD exhibit such global impairment in facial expression encoding. Sprengelmeyer et $\mathrm{a}^{13}$ found that idiopathic PD patients can accurately process and discriminate the identity of faces but then fail to correctly process the emotional expression in later stages of the task. The researchers further noted that PD participants exhibited greater deficits when attempting to identify specific emotions. Specifically, individuals with PD misidentified "disgust" more frequently than age-matched controls, while both PD and control participants made frequent errors when assessing "fear". Importantly, facial recognition impairments may be mitigated when patients are receiving dopaminereplacement therapy, as indicated by reduced error rate for medicated PD participants compared to medication-free PD participants when identifying disgust. ${ }^{13}$ Somewhat consistent with the findings of Sprengelmeyer et al,,$^{13}$ Suzuki et a ${ }^{14}$ found an emotion recognition deficit for participants in the early stages of idiopathic PD, all of whom were receiving dopamine replacement therapy, that was specific to disgust.

Evidence from early studies investigating emotion identification impairment in PD emphasized the need for clarification when nuances, such as medication status, were considered. In response to this call for integration and clarification, Gray and Tickle-Degnen ${ }^{15}$ reviewed a multitude of studies on emotion recognition in PD. Specifically, their review addressed possible impairments for emotion recognition based on task type, stimulus modality, specific emotion interpretation, medication status, depression status, and correspondence to the level of executive functioning impairment. The results indicated that the effect of PD on emotion recognition as a function of stimulus modality was more severe when interpreting prosodic expression compared to facial expressions. ${ }^{15}$ The moderating role of task type was investigated by comparing performance on three tasks: emotion identification, emotion discrimination, and a rating task. Findings indicated that individuals with PD show the greatest error rate when assessed via an identification or discrimination task for both facial and prosodic expression measures. ${ }^{15}$ Both of these emotion recognition tasks require participants to categorize the intended meaning of either a facial or a prosodic expression; however, an identification task requires the additional demand of producing a semantic label. Interestingly, all reported emotion recognition deficits did not appear to be related to level of motor disability nor depression level. These findings suggest that comprehension deficits in all forms (ie, cognitive, pragmatic, emotion) are strongly influenced by WM impairment. ${ }^{15}$

Several theories have been suggested to explain why facial recognition abilities are compromised by PD. The simulation theory suggests that the constructive and unconscious tendency for a perceiver to mimic the emotional expression of the interlocutor is what allows us to figuratively place ourselves in another person's situation. ${ }^{16}$ Through a process known as facial feedback, the spontaneous mimicry of a partner's emotional state in one's own facial expression allows the listener to gain insight into the proper classification of the speaker's true emotional state by simulating the affective component of the speaker's perspective. As PD commonly results in a decline in the ability to express emotions via the face, the theory proposes that it is the inability of individuals with PD to properly mimic the speaker's displayed emotion that results in impaired emotion awareness. ${ }^{17}$ Though this theory accounts for an affective component seemingly essential to accurate emotion recognition, it has not been clearly established that to recognize an emotional state, one must also be able to express that state, thus the theory lacks an equally essential cognitive component.

Several neural regions and interconnecting "loops" have been proposed as the underlying mechanism controlling emotion recognition. Alexander et a ${ }^{18}$ proposed that the neurophysiology of the basal ganglia consists of three separable loops, which serve to connect the putamen, the caudate nucleus, and the nucleus accumbens with several cortical and subcortical regions. The circuit contains the critical 
nigrostriatal and mesolimbic dopaminergic systems, which conjointly function in modulating information processing within the striatum. As each of the three loops differentially connects and affects distinct brain areas, Alexander et $\mathrm{al}^{18}$ hypothesized that a specific pattern of motor, cognitive, and emotional impairment can be anticipated. Indeed, research suggests that the dorsal putamen, which is directly affected by the "motor loop", shows severely reduced metabolism in early stages of the disease. Reduced functionality in the motor loop can result in reduced control over movement preparation, movement direction, and the velocity and amplitude of movement. ${ }^{13}$ Evidence further suggests that as PD progresses, the dorsal caudate nucleus, which receives projections from the "complex loop", and the posterior parts of the ventral putamen, associated with the "limbic loop", also show significant metabolism reduction. Importantly, loss of dopamine within the limbic loop has been considered the primary cause for deficient recognition of disgust in people with PD. As previously discussed, Sprengelmeyer et $\mathrm{a}^{13}$ provided strong evidence of right ventral putamen activation in parallel to viewing facial expressions of disgust.

\section{Pragmatic meaning comprehension deficits in PD}

Pragmatic meaning comprehension refers to the ability to understand what a speaker intends to convey with an utterance and is normally focused on nonliteral language such as metaphor, irony, and sarcasm. Successful comprehension requires the integration of multiple sources of information, including the social context, the speaker's emotional state, and so on.

Several studies within the past decade suggest that nondemented and medicated PD patients exhibit deficits in several nonliteral language domains. ${ }^{19-21}$ As some degree of shared understanding of a recipients' utterance is essential for successful language use, early deficits in this domain should be examined when determining early diagnosis and strongly considered when approaching conversations.

Monetta and Pell ${ }^{20}$ studied metaphor comprehension in a group of medicated PD patients and found that individuals with PD committed more metaphor interpretation errors and were slower to respond compared to control participants. Interestingly, metaphor comprehension was only impaired in PD participants who also demonstrated extreme deficits in WM. ${ }^{20}$ In a later work by Monetta et al, ${ }^{19}$ irony comprehension and lie detection were examined in a similar nondemented group of PD patients. Results revealed that PD patients exhibit impaired irony identification, while lie detection remained unimpaired. Consistent with the authors' previous findings, ${ }^{20}$ two assessments measuring verbal WM and fluency were strongly related to deficits in the lie detection story task. ${ }^{19}$

In addition to nonliteral language, pragmatic meaning comprehension can also refer to illocutionary force or what a speaker intends to achieve with an utterance. ${ }^{22}$ Holtgraves and McNamara ${ }^{21}$ investigated PD patients' speech act comprehension by having nondemented PD patients and age-matched controls perform a timed lexical decision task following an utterance that performed a specific speech act (ie, beg) or a matched utterance that did not perform that speech act. Participants in the control group exhibited the expected priming for the action verb after the speech act utterance. However, the PD participants did not exhibit this priming effect, suggesting that speech act activation is slowed or is no longer an automatic component of comprehension. ${ }^{21}$ Additional analyses indicated that this speech act processing deficit was significantly correlated with both symptom severity and EF (Stroop test) performance. ${ }^{21}$ In a follow-up study, the same participants read scenarios and utterances and were asked to provide a single word that they believed best described the action the speaker was performing. PD participants correctly identified significantly fewer speech acts than did the control participants, demonstrating a deficit in speech act recognition in PD that is independent of temporal constraints.

Lloyd $^{23}$ also studied illocutionary force in terms of receptive prosodic loss or the ability to comprehend behavioral and verbal nuances during conversation. Results indicated that nondemented PD patients who were not suffering from depression showed impaired ability to accurately identify utterance prosody and displayed deficits for utterance prosody comprehension. Such findings suggest that verbal and emotional nuances, such as a subtle change in tone of voice or facial expression, may go undetected by PD patients. ${ }^{23}$

Finally, Berg et $\mathrm{al}^{24}$ examined nonliteral language comprehension in PD patients without cognitive deficits. Though language comprehension was studied using a variety of subtasks, only inference accuracy and sentence analysis were significantly impaired in PD participants compared to controls. ${ }^{24}$ Understanding the degree to which pragmatic comprehension deficits impact overall understanding of everyday conversation is essential if language use is to be successful.

\section{Recommendations}

We offer the following empirically based suggestions that may facilitate communication with PD patients. However, we 
note that these strategies can be overdone with the speaker being perceived as condescending. Hence, judicious care should be taken when following these recommendations so as to lessen this risk. In addition, just as each individual's experience with PD is unique, utilization of these recommendations are subject to an individual's specific area(s) of impairment. Thus, physicians, caretakers, and PD patients should discuss particular circumstances in which these recommendations may best serve to facilitate communication.

1. Avoid complex syntax. Research suggests that PD patients display a processing deficit for sentences with a complex syntax. ${ }^{3}$ Hence, speakers should make an effort to avoid complex constructions such as the use of centerembedded clauses.

2. Avoid taxing WM. Research suggests that reducing the amount of information requiring maintenance in WM can diminish processing deficits in people with PD. ${ }^{4-6}$ In addition to simpler syntax, speakers are advised to actively ground their conversational contributions, ${ }^{25}$ that is, to chunk the information they are conveying into smaller units and wait for acknowledgment before proceeding. (Note that acknowledgment sometimes may need to be elicited from the individual.)

3. Clearly delineate topics. Conversations should be structured so as to thoroughly discuss a single subject in a compartmentalized manner before shifting to a new and unrelated topic. Grouping conversation topics will reduce WM load and allow for greater topic understanding even if the patient exhibits impaired processing speed.

Set shifting is another EF that has consistently been found to be impaired due to PD. ${ }^{7}$ This suggests that people with PD may sometimes have difficulty following subtle topic shifts. It is recommended, then, that speakers use topic markers (eg, "Returning to what we were talking about earlier...") and make it clear when transitioning to a new topic. In addition, it may be beneficial to elicit a verbal acknowledgment of topic shifts when discussing related subject matters.

4. Avoid reliance on emotional expressions. People are typically in the habit of relying on their emotional expressions (either facial expressions or emotional prosody) to allow others to disambiguate their meaning. However, individuals with PD show a deficit in recognizing others' emotional expressions, especially in terms of emotional prosody. ${ }^{15}$ People should be aware of this and not rely so much on emotional expressions to help disambiguate their utterances.

5. Avoid nonliteral meaning. When conversing with people with PD, there should be an attempt to convey meaning directly, so that recipients are not required to engage in extensive inferential processing in order to correctly recognize one's intended meaning. Furthermore, speakers should encourage patients to give feedback on the interpretation of the conveyed utterance to ensure the intended meaning was accurately received.

6. Use performative verbs. In addition to the recognition of nonliteral meaning, it appears that some individuals with PD may be impaired in terms of recognizing speech acts. ${ }^{21}$ One way to deal with this is to use performative verbs, that is, verbs that name the speech act that is being performed. Hence, to remind someone of something, use a form of the verb "remind" rather than leaving it implicit.

7. Minimize distractions. Conversations with PD patients will be enhanced if they are held in a quiet environment with minimal background distractions and the speaker is oriented to face the listener. Also, when speaking to individuals with $\mathrm{PD}$, the speaker should try to speak at an octave slightly above normal range and at a reduced rate, particularly when communicating complex information. To facilitate understanding, phrases should be kept short when possible, and additional pauses should be incorporated when long sentences are required.

\section{Production deficits}

Relative to research on comprehension deficits, there has been less research examining production deficits in PD. This imbalanced emphasis on comprehension deficits reflects the innate difficulty in disentangling the two communication domains. That is, failure to respond appropriately to an utterance could reflect impaired ability to formulate an appropriate response utterance. However, it is just as possible that an inappropriate response results from a failed attempt to comprehend the entirety of an utterance just provided by a speaker. Nevertheless, in this section, we review literature on possible language production deficits that result from PD and conclude with our recommendations on factors, which we hope will serve to minimize communication impairments.

\section{Reduced language production and informational content in PD}

Information has accumulated over the past several decades indicating a significant reduction in language production and informational content in utterances produced by individuals with PD. In an early study, Cummings ${ }^{26}$ examined language production in patients with $\mathrm{PD}$ (both with and without various types of dementia). Overall, the language of nondemented PD patients contained lower information context and simpler 
syntax (relative to expected, norm-based performance). In a related language production study, Illes et $\mathrm{al}^{27}$ supported Cummings ${ }^{26}$ findings of PD patients displaying lower syntactic complexity relative to control participants. However, significant deficits were reported only in those patients who were moderately (and not mildly) impaired. A possible explanation for this caveat was provided by the additional finding that syntactic complexity was strongly correlated with dysarthria severity and PD severity. ${ }^{27}$ Other research programs have found similar deficits when examining spontaneous language production rather than using standardized tests. For example, using a spontaneous task, Small et al ${ }^{28}$ found significant differences in sentence length, number of propositions, and grammatical complexity between nonimpaired controls and PD participants with moderate dementia; no significant language differences were found between the controls and nondemented and mildly demented PD participants. Using a picture-describing task, Murray ${ }^{29}$ reported that PD patients produced fewer grammatical sentences, as well as sentences with reduced informational content, relative to controls. A related study conducted by Murray and Lenz ${ }^{30}$ examined language production in conversational discourse and reported no syntactical impairment in the PD group, although the degree of dementia was negatively related to syntactic complexity and sentence length.

\section{Speech fluency abnormalities, inappropriate pauses, and impaired grammar in PD}

In addition to a reduction in utterance informational content $^{26,29}$ and an overall drop in speech production, ${ }^{28-30} \mathrm{PD}$ has also been associated with abnormalities in speech fluency in the form of prolonged and inappropriate pauses and incorrect verb usage. Illes ${ }^{31}$ examined spontaneous language production in patients with PD and found fluency disruption in the form of long-duration silent hesitations as well as openclass verbal paraphasia. However, in contrast to Cummings, ${ }^{26}$ PD patients in this study did not significantly differ from controls in terms of syntax. ${ }^{31}$ This finding was also demonstrated by Hammen and Yorkston ${ }^{32}$ who report that PD patients also demonstrated a tendency to pause inappropriately (eg, within a phrase or a clause) relative to controls.

Verbal fluency deficits in PD have been investigated frequently. Henry and Crawford ${ }^{33}$ conducted a meta-analysis and concluded that people with PD display significant impairment on measures of both semantic and phonetic fluency. In addition, the semantic fluency deficit appears to be significantly larger than the phonetic deficit and independent of cognitive speed and effortful retrieval, suggesting that semantic memory is particularly impaired in PD. Furthermore, the relative prominence of semantic to phonetic deficits in PD with dementia is a feature that serves to differentiate it from other types of dementia such as Alzheimer's type. Interestingly, Zanini et $\mathrm{al}^{34}$ examined sentence production in bilingual PD patients and found more grammatical errors for PD patients, relative to controls, but only for their first and not their second language. The authors suggest that participants' first language is more likely to reflect implicit, procedural processing and hence more likely to engage basal ganglia structures, which are impaired with PD. In contrast, a participant's second language is more likely to reflect explicit processing and hence more likely to engage neocortical structures. ${ }^{34}$

More recently, Troche and Altmann ${ }^{35}$ examined the correlation between cognitive deficits and language production in PD using sentence repetition and generation tasks. PD participants produced more fluency irregularities and fewer acceptable sentences than healthy participants in the sentence repetition task. For the sentence generation task, PD participants were impaired, relative to healthy participants, on fluency, grammaticality, and completeness, although the impairment was largest for fluency. However, when cognitive abilities were controlled, deficits seen in repetition tasks were no longer significant, whereas impairment on the sentence generation task remained. ${ }^{36}$ Finally, Coleman et $\mathrm{al}^{37}$ reported a more specific grammatical impairment in PD, a deficit in terms of producing a correct verb form, whereas noun production was not impaired.

In sum, there is considerable evidence for a cluster of specific language production deficits in $\mathrm{PD}$, including lowered informational content, speech fluency abnormalities, long and inappropriate pauses, and impaired grammar, all of which can influence one's ability to contribute appropriately to a conversation. These deficits are not necessarily independent. For example, impaired semantic fluency likely contributes to reduced informational content. Also, some of these production deficits (eg, lowered informational content) may reflect a comprehension deficit (failure to understand a prior turn) rather than a production deficit per se. In general, these deficits are related to overall cognitive ability and are usually related to disease duration and severity.

\section{Respiratory physiologic issues and production deficits in PD}

Successful speech production relies not only on language planning and cognitive resources but also on the capacity to perform proper motor plans and movements of the respiratory, laryngeal, and supralaryngeal systems. Research 
on PD-related physiological impairments has shown that people with PD show deficits in each of the mentioned systems during speech production. For example, Huber and Darling ${ }^{38}$ found that PD patients produced shorter utterances compared to control participants and that this reduction was directly related to decreased lung volume initiation and weaker inspiratory duration in the PD group. Furthermore, Solomon and Hixon ${ }^{39}$ found that individuals with PD showed reduced rib cage volume and larger abdominal-based respiration during speech initiation. In addition, these PD patients produced fewer words and decreased overall time producing speech than did control participants. ${ }^{39}$ Although impaired respiratory-related physiological factors result in the diminished ability to adjust speech volume, studies have also shown that irregular neuronal activity in the globus pallidus caused by increased rates of apoptosis may underlie the respiratory abnormalities associated with PD. ${ }^{40,41}$

Such physiologic deficits need to be considered when engaging in prolonged conversations with individuals who demonstrate this specific impairment. Further emphasizing the need for increased attention to respiratory system deficiency in PD populations, Ho et $\mathrm{al}^{42}$ investigated the irregularities in speech volume control (hypophonia) caused by $\mathrm{PD}$ and its significant impact on overall satisfaction gained through human interaction, and specifically conversation. The first two experiments examined the impact of PD on an individual's ability to automatically regulate speech volume when background noise and instantaneous auditory feedback were manipulated. Results showed that compared to controls, PD participants were less able to increase their speech volume when background noise increased and similarly showed reduced tendencies to decrease volume as instantaneous auditory feedback increased. ${ }^{42}$ These findings illustrate that individuals with PD tend to demonstrate overconstancy with speech volume and thus fail to accurately respond to contextually appropriate implicit cues. However, in the third experiment carried out by $\mathrm{Ho}$ et $\mathrm{al},{ }^{43}$ individuals with $\mathrm{PD}$ were verbally instructed to increase/decrease their speech volume to determine if participants previously failing to respond to subtle context cues were capable of appropriately modulating their speech volumes. Findings showed that when verbally instructed to do so, PD participants expressed normal fluctuations in volume and thus maintained the capacity to vary vocal range. ${ }^{43}$

Some researchers have examined how the speech of individuals with PD influences the manner in which they are perceived. Pitcairn et $\mathrm{al}^{44}$ had participants listen to semi-structured interviews with either PD or unimpaired speakers. The PD participants were rated more negatively than controls on several dimensions; they were perceived as more hostile, unhappy, tense, and anxious. Acoustically, PD individuals displayed less pitch variation and more pauses than the controls. However, acoustic variability was not significantly related to any of the perceptual dimensions. ${ }^{44}$

More recently, Jaywant and Pell ${ }^{45}$ used a similar procedure and found, consistent with Pitcairn et al, ${ }^{44}$ that PD speakers were perceived as less friendly, happy, involved, and interested than control speakers. Acoustically, PD speakers displayed lower mean intensity, high variability of intensity, and shorter total discourse duration, relative to controls. ${ }^{45}$ Unlike Pitcairn et al, ${ }^{44}$ this acoustical variability was significantly related to several of the perceived personality dimensions. In terms of perceptions of the content of talk, there was an unexpected finding for the content of the PD speakers' talk to be perceived more positively (eg, coherent, comprehensible, interesting) than that of the control participants' talk. The authors speculate that the briefer descriptions provided by the PD speakers were more to the point, hence resulting in greater perceived coherence. In any event, the authors emphasize the negative consequences of PD speech in that they tend to be perceived as less friendly, happy, and involved. ${ }^{45}$

\section{Pragmatic production deficits in PD}

An early report examining pragmatic production deficits in PD was conducted by McNamara and Durso, ${ }^{46}$ in which, participants (PD and control) engaged in brief conversations with a member of the research team. The conversations were coded using the pragmatic checklist of Prutting and Kirchner $^{47}$, a scheme for classifying social language skills in terms of verbal features (eg, topic selection, topic maintenance, turn taking, lexical variation), paralinguistic features (prosody, vocal quality), and nonverbal features (eg, gaze, gestures). PD participants were impaired on $20.4 \%$ of the items relative to the control participants. However, the PD and control participants did not vary significantly on measures of mental status or verbal fluency (although the scores of the former were lower than the latter on both), suggesting that the pragmatic impairment was not simply reducible to global cognitive deficits or poverty of speech. ${ }^{46}$ At the same time, there were significant $\mathrm{PD}$-control differences on the performance of tasks related to frontal lobe deficits (Stroop test and design fluency), and for the PD participants, these measures were significantly correlated with their pragmatic performance. ${ }^{46}$ In a separate study, PD participants were asked to rate themselves on dimensions derived from the protocol of Prutting and Kirchner, ${ }^{47}$ and their spouse or significant other also rated 
them using the same items. The PD participants consistently overestimated their pragmatic abilities, relative to the ratings provided by their spouse or significant other, differences that were significant for speech acts, lexical selection, stylistics, and conversational appropriateness.

A similar study was conducted more recently by Hall et $\mathrm{al}^{48}$ using the Rehabilitation Institute of Chicago's Rating Scale of Pragmatic Communication Skills, ${ }^{49}$ a scale that evaluates ten categories of pragmatic function, four nonverbal dimensions (intonation, eye contact, facial expressions, gestures and proxemics) and six verbal dimensions (conversational initiation, turn-taking, topic maintenance, response length, presupposition, referencing skills). PD participants were significantly impaired overall, and the impairment was greater for nonverbal dimensions than for verbal dimensions. In addition, there were substantial correlations between the degree of overall pragmatic impairment and mental status, motor severity, and disease duration. ${ }^{48}$

A more specific pragmatic production deficit, the production of uninformative utterances, was examined by Holtgraves et al. ${ }^{50} \mathrm{PD}$ and healthy control participants' were interviewed regarding family, work history, daily activities, and so on. These interviews were taped and later coded by two raters (blind to group membership) in terms of degree of utterance informativeness. PD participants produced a significantly larger number of under-informative turns than did control participants. Importantly, the degree of informativeness was significantly correlated with automatic speech act recognition (as reported in Holtgraves and $\mathrm{McNamara}{ }^{21}$ ). That is, the less PD participants were able to recognize the intention behind another's remark, the greater their degree of under-informativeness in the interview. ${ }^{50}$

One promising approach to pragmatic production deficits in PD is politeness theory. ${ }^{51}$ In this approach, language users are assumed to be attentive to situation-based interpersonal considerations and structure their utterances so as to be responsive, to varying degrees, to these considerations. Holtgraves and McNamara ${ }^{21}$ used politeness theory to examine the possibility that individuals with PD might suffer a deficit in terms of conversational appropriateness. Using a role-playing methodology, PD and healthy controls were asked to indicate what they would say in various situations in which request imposition and power were manipulated. In general, PD participants produced lower levels of politeness and, more importantly, were less sensitive to variations in request size than were healthy controls; the former failed to modulate the politeness of their requests as a function of request size to the extent that the control participants did.
Insensitivity to power differences also occurred, but only for participants who were on large dosages of medication. Specifically, PD participants who were on higher dosage levels, relative to PD participants on lower dosage levels and control participants, did not vary their politeness as a function of the recipient's power. ${ }^{21}$

More recently, researchers have begun to analyze the manner in which individuals handle some of the pragmatic difficulties that may occur when interacting with PD patients. Considerable complexity emerges when an interacting dyad is treated as a system, and PD communication deficits can impact that system in multiple and complex ways. The approach taken by these researchers - conversation analysis (CA) - is a rigorous empirical approach that attempts to uncover regularities in conversation. ${ }^{52}$ Although there is a substantial CA literature, attempts to use this methodology in the examination of pragmatic difficulties in PD are recent and rare.

Saldert et $\mathrm{al}^{53}$ and Griffiths et $\mathrm{al}^{54}$ examined in detail naturally occurring conversations between PD patients and nonimpaired others (Griffiths et $\mathrm{al}^{55}$ for a more general discussion on the use of CA for analyzing PD interactional difficulties). These interactions were recorded and analyzed using common CA techniques. Several consistent patterns emerged. First, Griffiths et $\mathrm{al}^{54}$ focused on talk overlap. Although overlapping talk occurs in conversation, it is relatively infrequent and typically handled quickly and efficiently with repair (ie, attempts to correct) sequences. ${ }^{56}$ But timing is critical for these sequences to function, and since individuals with PD produce more silent pauses, ${ }^{31}$ the integrity of this system can be undermined. Also, as has been demonstrated, there is a strong systematic preference for self-repair over other-repair. ${ }^{57}$ However, PD speakers, due to both speech production (dysarthria) and cognitive deficits, are at a disadvantage in initiating repair sequences. And, in fact, this difficulty appears to frequently result in the deletion of PD turns at talk. Saldert et $\mathrm{al}^{53}$ focused primarily on trouble sources in conversations with PD participants. An important contribution of their work was the identification of cognitive difficulties as contributing to the initiation of repair sequences. Specifically, $70 \%$ of the instances of other-repair in these conversations were related to the semantic content of a turn produced by the individual with PD, typically wordfinding difficulty or use of atypical wording. The approach taken by these researchers is extremely important because it points specifically to the interactional difficulties that may occur in PD and how speech and cognitive deficits associated with PD may be magnified in interactional settings. 


\section{Recommendations}

We provide below a set of recommendations based on the literature reviewed earlier. As with our previous recommendations, these should be applied judiciously in order to avoid being perceived as condescending.

1. Do not judge. Individuals with PD display a variety of language production deficits (long and inappropriate pauses, reduced information content, impoliteness) that may result in negative perceptions. Awareness that these features may be part of the symptomatology resulting from this disorder is helpful in avoiding these unwarranted perceptions. Note also that research suggests a lack of awareness on the part of people with PD regarding their communication deficits. ${ }^{58}$

2. Be patient/do not rush. One of the clearest PD production deficits is the occurrence of long and sometimes inappropriate pauses. ${ }^{27}$ These can be problematic because of the critical role played by pauses in the management of conversational turn-taking. When interacting with PD individuals, it may be helpful to maintain awareness of their tendency to pause and give them time to complete any turns before initiating one's own turn.

3. Ask questions regarding utterance comprehension. Another relatively clear deficit is lowered informational content, ${ }^{29}$ a deficit that may reflect misunderstanding of the prior turn. Rather than taking a brief or incomplete utterance at face value, it may be sometimes useful to ask questions in an attempt to ascertain their current understanding of the conversation.

4. Ask simple questions. Because of the production deficits that are associated with PD, successful communication is more likely if questions addressed to PD patients are straightforward and require no extensive elaboration in response. When possible, ask questions that require a simple yes or no response.

5. Be understanding and encouraging. Encourage the person to speak louder when their speech volume is abnormally soft. This should facilitate comprehension and reduce the need to have the PD patient repeat statements and increase respiratory and cognitive demands. Also, as noted earlier, important conversations should be held in settings that are quiet and offer little distractions.

\section{Conclusion}

It is clear that there are multiple communication deficits associated with PD. The purpose of this paper was to organize and summarize existing empirical research on these deficits and provide recommendations for dealing with these deficits in order to facilitate communication with those who suffer from PD. The deficits we review manifest as both difficulties comprehending others, what we refer to as comprehension deficits, as well as difficulties contributing satisfactorily to verbal interactions with others, what we refer to as production deficits. Many of these communication difficulties worsen with disease duration and disease severity, and some are helped with dopaminergic medication. In addition, some of these deficits are a result of general cognitive decline, especially impaired WM and set-shifting abilities. It is important to realize that people suffering with PD may not be aware of their deficits. In general, we recommend awareness and sensitivity to these communication deficits, an approach that can contribute to more satisfactory and informative interactions.

\section{Disclosure}

The authors report no conflicts of interest in this work.

\section{References}

1. de Lau LM, Breteler MM. Epidemiology of Parkinson's disease. Lancet Neurol. 2006;5(6):525-535.

2. Garcia-Ruiz PJ, Chaudhuri KR, Martinez-Martin P. Non-motor symptoms of Parkinson's disease A review... from the past. J Neurol Sci. 2014;338(1):30-33.

3. Friederici AD, Kotz SA, Werheid K, Hein G, von Cramon DY. Syntactic comprehension in Parkinson's disease: investigating early automatic and late integrational processes using event-related brain potentials. Neuropsychology. 2003;17(1):133.

4. Grossman M, Carvell S, Gollomp S, et al. Cognitive and physiological substrates of impaired sentence processing in Parkinson's disease. J Cogn Neurosci. 1993;5(4):480-498.

5. Grossman M, Zurif E, Lee C, et al. Information processing speed and sentence comprehension in Parkinson's disease. Neuropsychology. 2002;16(2):174.

6. Grossman M, Cooke A, DeVita C, et al. Grammatical and resource components of sentence processing in Parkinson's disease: an fMRI study. Neurology. 2003;60(5):775-781.

7. Dirnberger G, Jahanshahi M. Executive dysfunction in Parkinson's disease: a review. J Neuropsychol. 2013;7(2):193-224.

8. Brown R, Marsden C. An investigation of the phenomenon of "set" in Parkinson's disease. Mov Disord. 1988;3(2):152-161.

9. Brown R, Marsden C. Internal versus external cues and the control of attention in Parkinson's disease. Brain. 1988;111(Pt 2):323-345.

10. Owen A, James M, Leigh P, et al. Fronto-striatal cognitive deficits at different stages of Parkinson's disease. Brain. 1992;115(Pt 6): 1727-1751.

11. Ariatti A, Benuzzi F, Nichelli P. Recognition of emotions from visual and prosodic cues in Parkinson's disease. Neurol Sci. 2008; 29(4):219-227.

12. Dewick HC, Hanley JR, Davies AD, Playfer J, Turnbull C. Perception and memory for faces in Parkinson's disease. Neuropsychologia. 1991; 29(8):785-802.

13. Sprengelmeyer R, Young AW, Mahn K, et al. Facial expression recognition in people with medicated and unmedicated Parkinson's disease. Neuropsychologia. 2003;41(8):1047-1057.

14. Suzuki A, Hoshino T, Shigemasu K, Kawamura M. Disgust-specific impairment of facial expression recognition in Parkinson's disease. Brain. 2006;129(Pt 3):707-717. 
15. Gray HM, Tickle-Degnen L. A meta-analysis of performance on emotion recognition tasks in Parkinson's disease. Neuropsychology. 2010;24(2):176-191.

16. Bodden ME, Mollenhauer B, Trenkwalder C, et al. Affective and cognitive theory of mind in patients with Parkinson's disease. Parkinsonism Relat Disord. 2010;16(7):466-470.

17. Freedman M, Stuss DT. Theory of mind in Parkinson's disease. J Neurol Sci. 2011;310(1-2):225-227.

18. Alexander GE, DeLong MR, Strick PL. Parallel organization of functionally segregated circuits linking basal ganglia and cortex. Annu Rev Neurosci. 1986;9(1):357-381.

19. Monetta L, Grindrod CM, Pell MD. Irony comprehension and theory of mind deficits in patients with Parkinson's disease. Cortex. 2009;45(8):972-981.

20. Monetta L, Pell MD. Effects of verbal working memory deficits on metaphor comprehension in patients with Parkinson's disease. Brain Lang. 2007;101(1):80-89.

21. Holtgraves T, McNamara P. Pragmatic comprehension deficit in Parkinson's disease. J Clin Exp Neuropsychol. 2010;32(4):388-397.

22. Searle JR. Speech Acts: An Essay in the Philosophy of Language. London: Cambridge University Press; 1969.

23. Lloyd AJ. Comprehension of prosody in Parkinson's disease. Cortex. 1999;35(3):389-402.

24. Berg E, Bjornram C, Hartelius L, Laakso K, Johnels B. High-level language difficulties in Parkinson's disease. Clin Linguist Phon. 2003;17(1):63-80.

25. Clark C, Moss PA, Goering S, et al. Collaboration as dialogue: teachers and researchers engaged in conversation and professional development. Am Educ Res J. 1996;33(1):193-231.

26. Cummings JL. Intellectual impairment in Parkinson's disease: clinical, pathologic, and biochemical correlates. J Geriatr Psychiatry Neurol. 1988;1(1):24-36.

27. Illes J, Metter E, Hanson W, Iritani S. Language production in Parkinson's disease: acoustic and linguistic considerations. Brain Lang. 1988;33(1):146-160.

28. Small JA, Lyons K, Kemper S. Grammatical abilities in Parkinson's disease: evidence from written sentences. Neuropsychologia. 1997; 35(12):1571-1576.

29. Murray LL. Spoken language production in Huntington's and Parkinson's diseases. J Speech Lang Hear Res. 2000;43(6):1350-1366.

30. Murray LL, Lenz LP. Productive syntax abilities in Huntington's and Parkinson's diseases. Brain Cogn. 2001;46(1-2):213-219.

31. Illes J. Neurolinguistic features of spontaneous language production dissociate three forms of neurodegenerative disease: Alzheimer's, Huntington's, and Parkinson's. Brain Lang. 1989;37(4):628-642.

32. Hammen VL, Yorkston KM. Speech and pause characteristics following speech rate reduction in hypokinetic dysarthria. J Commun Disord. 1996; 29(6):429-445.

33. Henry JD, Crawford JR. Verbal fluency deficits in Parkinson's disease: a meta-analysis. J Int Neuropsychol Soc. 2004;10(04):608-622.

34. Zanini S, Tavano A, Fabbro F. Spontaneous language production in bilingual Parkinson's disease patients: evidence of greater phonological, morphological and syntactic impairments in native language. Brain Lang. 2010;113(2):84-89.

35. Troche MS, Altmann LJ. Sentence production in Parkinson disease: effects of conceptual and task complexity. Appl Psycholinguist. 2012;33(02):225-251.

36. Troche MS, Altmann LJ. Sentence production in Parkinson disease: effects of conceptual and task complexity. Appl Psycholinguist. 2011;33(2):225

37. Coleman MR, Davis MH, Rodd JM, et al. Towards the routine use of brain imaging to aid the clinical diagnosis of disorders of consciousness. Brain. 2009;132(Pt 9):2541-2552.
38. Huber JE, Darling M. Effect of Parkinson's disease on the production of structured and unstructured speaking tasks: respiratory physiologic and linguistic considerations. J Speech Lang Hear Res. 2011;54(1):33-46.

39. Solomon NP, Hixon TJ. Speech breathing in Parkinson's disease. J Speech Hear Res. 1993;36(2):294-310.

40. Brotchie P, Iansek R, Horne MK. Motor function of the monkey globus pallidus. 1. Neuronal discharge and parameters of movement. Brain. 1991;114(Pt 4):1667-1683.

41. Brotchie P, Iansek R, Horne MK. Motor function of the monkey globus pallidus. 2. Cognitive aspects of movement and phasic neuronal activity. Brain. 1991;114(Pt 4):1685-1702.

42. Ho AK, Bradshaw JL, Iansek R, Alfredson R. Speech volume regulation in Parkinson's disease: effects of implicit cues and explicit instructions. Neuropsychologia. 1999;37(13):1453-1460.

43. Ho AK, Iansek R, Marigliani C, Bradshaw JL, Gates S. Speech impairment in a large sample of patients with Parkinson's disease. Behav Neurol. 1999;11(3):131-137.

44. Pitcairn TK, Clemie S, Gray JM, Pentland B. Non-verbal cues in the self-presentation of Parkinsonian patients. Br J Clin Psychol. 1990; 29(Pt 2):177-184.

45. Jaywant A, Pell MD. Listener impressions of speakers with Parkinson's disease. J Int Neuropsychol Soc. 2010;16(01):49-57.

46. McNamara P, Durso R. Pragmatic communication skills in patients with Parkinson's disease. Brain Lang. 2003;84(3):414-423.

47. Prutting CA, Kittchner DM. A clinical appraisal of the pragmatic aspects of language. J Speech Hear Disord. 1987;52(2):105-119.

48. Hall D, Ouyang B, Lonnquist E, Newcombe J. Pragmatic communication is impaired in Parkinson disease. Int J Neurosci. 2011;121(5): 254-256.

49. Halper AS, Cherney LR, Burns MS, Mogil SI. RIC evaluation of communication problems in right hemisphere dysfunction-revised (RICE-R). In: Halper AS, Cherney LR, Burns MS, editors. Clinical Management of Right Hemisphere Damage. Gaithersburg, MD: Aspen; 1996:99-132.

50. Holtgraves T, Fogle K, Marsh L. Pragmatic language production deficits in Parkinson's disease. Adv Parkinson's Dis. 2013;2:31-36.

51. Brown P, Levinson SC. Politeness: Some Universals in Language Usage. Vol. 4. Cambridge: Cambridge University Press; 1987.

52. Hutchby I, Wooffitt R. Conversation Analysis. Cambridge: Polity; 2008 .

53. Saldert C, Ferm U, Bloch S. Semantic trouble sources and their repair in conversations affected by Parkinson's disease. Int J Lang Commun Disord. 2014;49(6):710-721.

54. Griffiths S, Barnes R, Britten N, Wilkinson R. Potential causes and consequences of overlap in talk between speakers with Parkinson's disease and their familiar conversation partners. Semin Speech Lang. 2012;33(1):27-43.

55. Griffiths S, Barnes R, Britten N, Wilkinson R. Investigating interactional competencies in Parkinson's disease: the potential benefits of a conversation analytic approach. Int J Lang Commun Disord. 2011;46(5):497-509.

56. Sacks H, Schegloff EA, Jefferson G. A simplest systematics for the organization of turn-taking for conversation. Language. 1974; 50(4):696-735

57. Levinson SC. Pragmatics (Cambridge Textbooks in Linguistics). Cambridge: Cambridge University Press; 1983.

58. Seltzer B, Vasterling JJ, Mathias CW, Brennan A. Clinical and neuropsychological correlates of impaired awareness of deficits in Alzheimer disease and Parkinson disease: a comparative study. Neuropsychiatry Neuropsychol Behav Neurol. 2001;14(2):122-129. 
Journal of Parkinsonism \& Restless Legs Syndrome

\section{Publish your work in this journal}

Journal of Parkinsonism and Restless Legs Syndrome is an online, open access, peer-reviewed journal. The journal publishes review articles, historical reviews, original research articles, case reports, letters to the editor, clinical teaching cases, neuroradiology highlights, neuropathology highlights, neuropsychiatry highlights, autobiographies, conference

\section{Dovepress}

proceedings, abstracts and book reviews. The manuscript management system is completely online and includes a very quick and fair peerreview system, which is all easy to use. Visit http://www.dovepress.com/ testimonials.php to read real quotes from published authors.

Submit your manuscript here: http://www.dovepress.com/journal-of-parkinsonism--restless-legs-syndrome-journal 